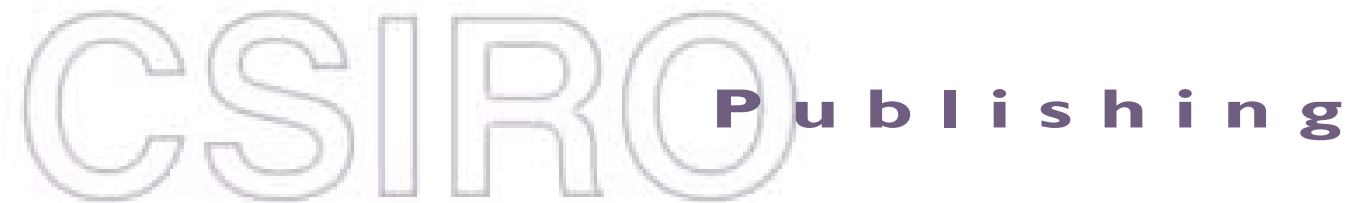

\section{Publications of the Astronomical Society of Australia}

Volume 19, 2002

(C) Astronomical Society of Australia 2002

An international journal of astronomy and astrophysics

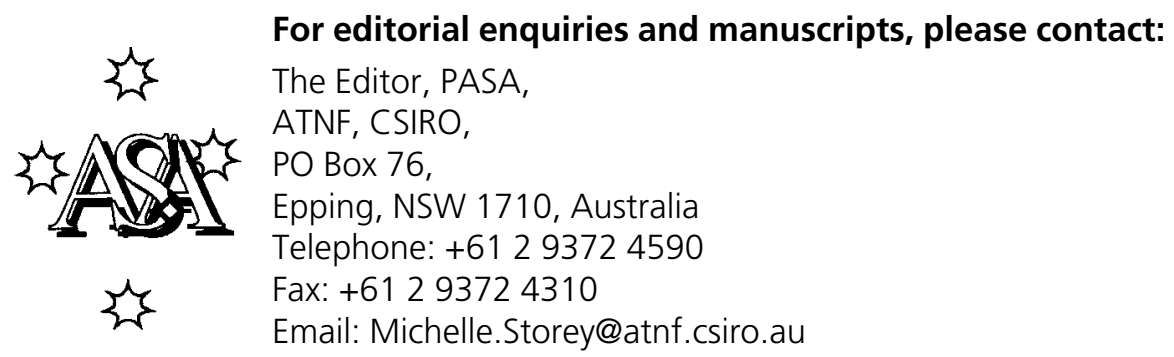

For general enquiries and subscriptions, please contact: CSIRO Publishing PO Box 1139 (150 Oxford St)

Collingwood, Vic. 3066, Australia

Telephone: +6139662 7666

Fax: +61 396627555

Email: publishing.pasa@csiro.au

C S I RO

PUBLISHING Published by CSIRO Publishing

for the Astronomical Society of Australia

www.publish.csiro.au/journals/pasa 


\title{
Ubiquitous Column Density Variability in Seyfert 2 Galaxies
}

\author{
G. Risaliti ${ }^{1,2}$, M. Elvis $^{2}$ and F. Nicastro ${ }^{2}$ \\ ${ }^{1}$ Ossevatorio Astrofisico di Arcetri, Largo E. Fermi, 5, I-50125 Firenze, Italy \\ risaliti@arcetri.astro.it \\ ${ }^{2}$ Harvard-Smithsonian Center for Astrophysics, 60 Garden Street, Cambridge, MA 02138, USA \\ Received 2001 August 4, accepted 2002 April 7
}

\begin{abstract}
We present a study of X-ray column density variability in Seyfert 2 galaxies. We show that variations in $\mathrm{N}_{H}$ are observed in almost all the objects with multiple hard X-ray observations. Variation timescales (as short as a few months in several cases) are not in agreement with the standard scenario of a parsec-scale toroidal absorber. We propose that the X-ray absorber in Seyfert galaxies is located much nearer to the centre than previously assumed, on the broad line region scale. An extension of the model by $\mathrm{M}$. Elvis (2000) can explain the observed variability. We also show preliminary results of $\mathrm{N}_{H}$ variability search inside single $\mathrm{X}$-ray observations, which suggest that variations can occur on timescales of a few $10^{4} \mathrm{~s}$.
\end{abstract}

Keywords: galaxies: active - galaxies: Seyfert $-\mathrm{X}$-rays: galaxies

\section{Introduction}

Strong obscuration is observed in the hard $(2-10 \mathrm{keV})$ $\mathrm{X}$-ray spectra of Type 2 active galactic nuclei (AGN), where a photoelectric cutoff at energies $\mathrm{E}>1-2 \mathrm{keV}$ indicates the presence of a column density of absorbing gas $\mathrm{N}_{H}>10^{22} \mathrm{~cm}^{-2}$.

The simplest geometry for this gas surrounding the nucleus is that of a torus covering $\sim 80 \%$ of the solid angle (in order to reproduce the 4:1 observed ratio between unobscured and obscured AGN, Maiolino \& Rieke 1995). One of the unsolved questions about this putative torus is its typical dimensions. Detailed models have been proposed for both a 100 parsec-scale torus (Granato, Danese, \& Franceschini 1997) and for a parsec-scale one (Pier \& Krolik 1992). Both models are supported by observational evidence, so it is likely that both the components could be present in AGN. Here we investigate the variability of the $\mathrm{X}$-ray absorbing column density in X-ray defined Seyfert 2s having column densities higher than $\sim 10^{22} \mathrm{~cm}^{-2}$, but less than $10^{24} \mathrm{~cm}^{-2}$ (in order to have a measurement of the photoelectric cutoff in the $2-10 \mathrm{keV}$ band). We collected all the data available in the literature for Seyfert $2 \mathrm{~s}$ and we complemented them with the analysis of unpublished data in the ASCA and BeppoSAX public archives. In the following sections we show the results and we show that they can be explained within a consistent physical picture only assuming that the absorber is located at a distance from the centre typical of the broad emission line region (BELR).

\section{Results}

We found that a sample of 25 sources were observed at least twice in hard X-rays. Out of these 25 sources, 22 show $\mathrm{N}_{H}$ variability on timescales from a few months to years (Figure 1). The full detailed analysis of the 139

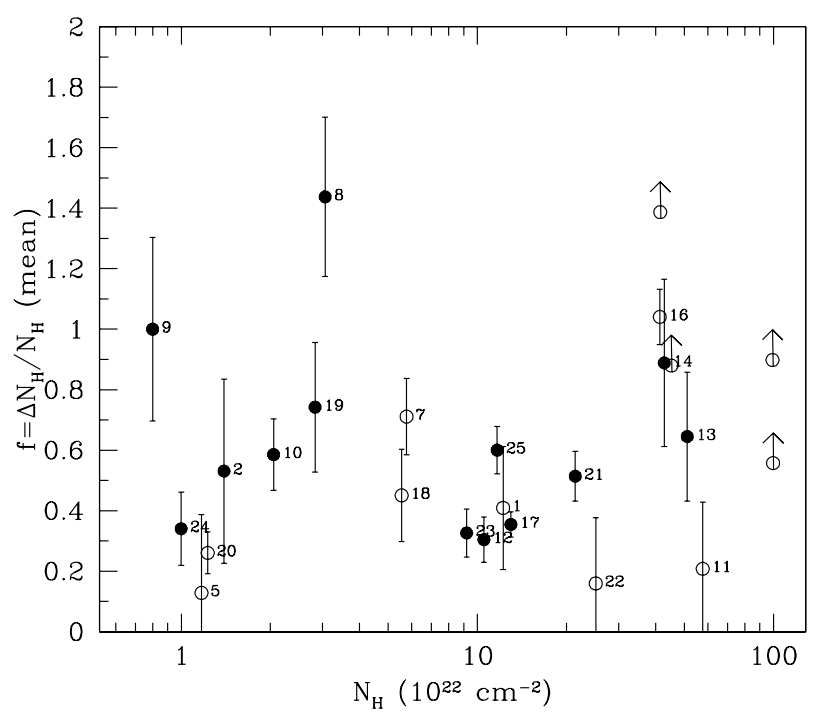

Figure 1 Ratio between column density variations $\Delta \mathrm{N}_{H}$ and mean $\mathrm{N}_{H}$, versus mean $\mathrm{N}_{H}$ for all the Seyfert $2 \mathrm{~s}$ with multiple hard X-ray observations. $\Delta \mathrm{N}_{H}$ is defined as the difference between the two measurements of $\mathrm{N}_{H}$. When more than two $\mathrm{N}_{H}$ measurements are available, we choose the two with the statistically most significant variation. Empty circles are for sources observed twice, filled circles are for sources with three or more observations.

observations of these 25 sources is described in Risaliti et al. (2002).

There are two physical reasons that can explain the variability of the absorbing column density: variation in the ionisation state of the absorber, due to variations in the ionising continuum, and variations of the amount of gas along the line of sight. We ruled out the first possibility, since the $\mathrm{N}_{H}$ variations are not correlated with the flux variations (see Risaliti et al. 2002 for details). The second scenario - motions in a clumpy medium - is the only one that can account for the observations. However, the results on the ubiquity of $\mathrm{N}_{H}$ variability in Seyfert $2 \mathrm{~s}$, together 
with the short (from two months to a few years) variability timescales, pose severe problems for the standard torus model. We can idealise the situation by assuming the typical timescale of variation, $t$, to be the crossing time of a discrete cloud across the line of sight. Assuming that the absorption is due to spherical clouds moving with Keplerian velocities, the distance from the central black hole of mass $M_{\bullet}$ is given by

$$
\begin{aligned}
R & \sim 3 \times 10^{16} \frac{M_{\bullet}}{10^{9} M_{\odot}}\left(\frac{\rho}{10^{6} \mathrm{~cm}^{-3}}\right)^{2} \\
& \times\left(\frac{t}{5 \mathrm{Msec}}\right)^{2}\left(\frac{\mathrm{N}_{H}}{10^{22} \mathrm{~cm}^{-2}}\right)^{-2} \mathrm{~cm}
\end{aligned}
$$

where $\rho$ is the density of the cloud. The black hole mass and the cloud density have been normalised to extreme values for a putative torus in order to obtain the greatest distance.

The distance we obtained is typical for the BELR. Even if many parameters are poorly constrained, it is not possible to obtain a value of $R>10^{18} \mathrm{~cm}$ within a consistent physical scenario. Therefore, the parsec-scale torus model is not able to explain our data. An alternative scenario, within the standard AGN model (Antonucci 1993), is that the X-ray absorber is located in the BELR, much nearer to the central black hole than the standard torus. If we assume that the broad line clouds are responsible for the absorption in the X-rays, we can find a consistent combination of the parameters in the previous equations, with higher cloud densities $\left(\rho \approx 10^{9} \mathrm{~cm}^{-3}\right)$ and shorter variability timescales $(t \approx 3$ days). We note that such timescales are not ruled out by our data, since we cannot investigate variations shorter than the time interval between two observations of the same source.

An absorber which is very compact (as required by our data) and axisymmetric (as required by the arguments supporting the unified schemes) can be easily obtained by extending the wind model by Elvis (2000, and these proceedings). In this model most of the phenomenology of Type 1 AGN is explained through a two-phase wind arising from the accretion disc. The cold phase of the wind is formed by the broad emission line clouds (BELC). A simple extension of this model could be that in type 2 AGN the wind is thicker, and the BELC cover all lines of sight through the wind, as illustrated in Figure 2. The external part of the wind can well be cold enough for dust to survive, therefore this absorber can also explain the optical properties of Seyfert 2s. Interestingly, the average dustto-gas ratio predicted by this model, assuming that the external part of the wind has a standard ISM composition, is lower than Galactic, in agreement with recent findings (Maiolino et al. 2001; Risaliti et al. 2001)

\section{Conclusions and Future Work}

Variability of X-ray absorbing column density appears to be an ubiquitous property in Seyfert 2 galaxies. Variation timescales can be as short as a few months. We have shown that these data rule out an X-ray absorber on a parsec-scale.

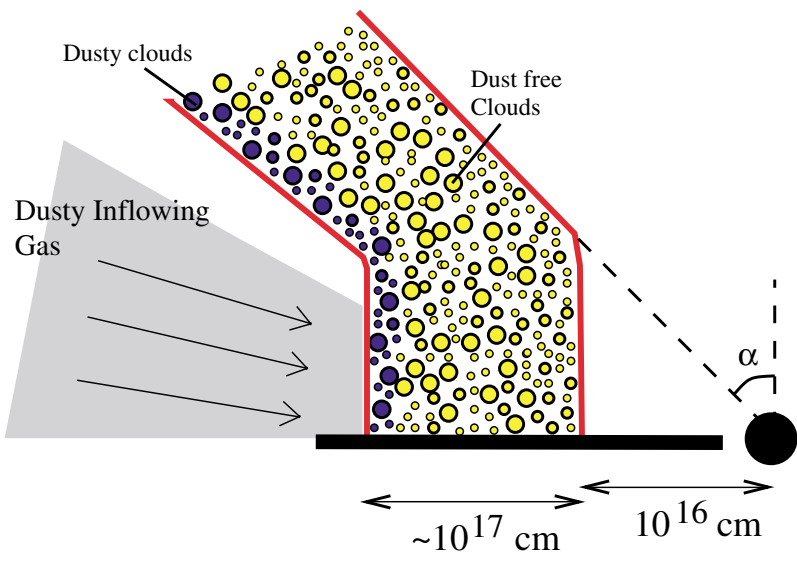

Figure 2 A simple model, derived from Elvis (2000), which explains the X-ray absorption properties of Seyfert 2s. The X-ray central emission is absorbed by the broad emission line clouds. The column density variability timescale is the average crossing time of a cloud along the line of sight. The covering factor of the funnel-shaped absorber (given by $1 \cos \alpha$ ) should be of the order of $0.7-0.8$, in order to reproduce the observed ratio between Type 2 and Type 1 AGN.

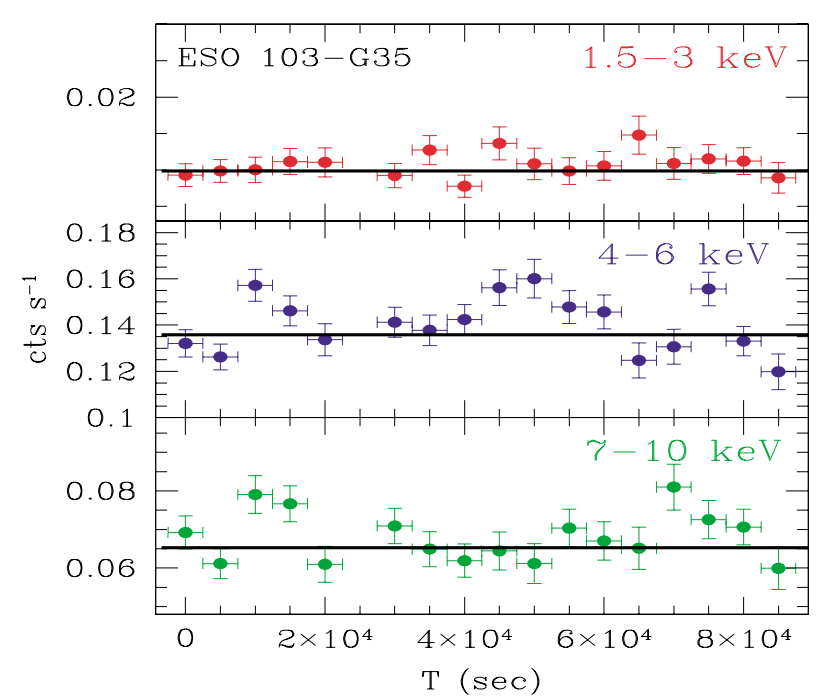

Figure 3 X-ray light curves of a BeppoSAX observation of ESO $103-\mathrm{G} 35$. The excess between 40 and $60 \mathrm{k}$ in the second is suggestive of an $\mathrm{N}_{H}$ variation. A subsequent spectral analysis has shown that this variation is significant at a $5 \sigma$ level of confidence.

Instead, we propose that the BELC in an axisymmetric distribution, as in the model of Elvis (2000), are responsible for X-ray absorption. If this is the case, variations in $\mathrm{N}_{H}$ are expected on timescales of days. Our variability study is limited by the shortest time between two observations of the same source. However, our work can be significantly improved looking for $\mathrm{N}_{H}$ variations inside single, long observations of the brightest sources in our sample. This work is still in progress. However we can show some preliminary, promising results. In Figure 3 we plot three light curves for three different energy intervals of a BeppoSAX observation of the Seyfert 2 ESO 103-G35.

The intervals are chosen is order to have the photoelectric cutoff at the separation energy between the first two light curves. Therefore, in the case of variations of $\mathrm{N}_{H}$, 
we expect that one of the first two light curves varies, while the other two remain constant (the third energy interval is little affected by small $\mathrm{N}_{H}$ variations). Instead, if we have a flux variation, we expect both the second and third light curve to vary, while the first remains constant, since the emission at energies lower that the cutoff is mainly due to an extended/reprocessed component. It is clear from Figure 3 that in the interval between 40 and $60 \mathrm{ks}$ the light curves vary in a way suggesting $\mathrm{N}_{H}$ variability. To check if this is indeed the case, we extracted two spectra, one in the 40-60 ks interval, and the other in the remaining time intervals. We performed a careful fit of these spectra, and concluded that the $\mathrm{N}_{H}$ measurements differ by $\sim 3 \times 10^{22} \mathrm{~cm}^{-2}$, at a $5 \sigma$ level of confidence. This $\mathrm{N}_{H}$ variation on a timescale of $\sim 20 \mathrm{ks}$ strongly suggests that the X-ray absorber is very close to the central black hole, in agreement with the model we have proposed.

\section{Acknowledgments}

This work was supported in part by NASA grant NAG54808 .

\section{References}

Antonucci, R. R. 1993, ARA\&A, 31, 473

Elvis, M. 2000, ApJ, 545, 63

Granato, G., Danese, L., \& Franceschini, A. 1997, ApJ, 486, 147

Maiolino, R., \& Rieke, G. H. 1995, ApJ, 454, 95

Maiolino, R., Marconi, A., Salvati, M., Risaliti, G., Severgnini, P., Oliva, E., La Franca, F., \& Vanzi, L. 2001, A\&A, 365, 28

Pier, E. A., \& Krolik, J. H. 1992, ApJ, 401, 99

Risaliti, G., Marconi, A., Maiolino, R., Salvati, M., \& Severgnini, P. 2001, A\&A, 371, 37

Risaliti, G., Elvis, M., \& Nicastro, F. 2002, ApJ, 571, 234 